\title{
The dynamical history of the evaporating or disrupted ice giant planet around white dwarf WD J0914+1914
}

\author{
Dimitri Veras ${ }^{\oplus 1,2 \star} \dagger$ and Jim Fuller ${ }^{3}$ \\ ${ }^{1}$ Centre for Exoplanets and Habitability, University of Warwick, Coventry CV4 7AL, UK \\ ${ }^{2}$ Department of Physics, University of Warwick, Coventry CV4 7AL, UK \\ ${ }^{3}$ TAPIR, Mailcode 350-17, California Institute of Technology, Pasadena, CA 91125, USA
}

Accepted 2020 January 29. Received 2020 January 27; in original form 2019 December 4

\begin{abstract}
Robust evidence of an ice giant planet shedding its atmosphere around the white dwarf WD J0914+1914 represents a milestone in exoplanetary science, allowing us to finally supplement our knowledge of white dwarf metal pollution, debris discs, and minor planets with the presence of a major planet. Here, we discuss the possible dynamical origins of this planet, WD J0914+1914 b. The very young cooling age of the host white dwarf (13 Myr) combined with the currently estimated planet-star separation of about 0.07 au imposes particularly intriguing and restrictive coupled constraints on its current orbit and its tidal dissipation characteristics. The planet must have been scattered from a distance of at least a few au to its current location, requiring the current or former presence of at least one more major planet in the system in the absence of a hidden binary companion. We show that WD J0914+1914 b could not have subsequently shrunk its orbit through chaotic f-mode tidal excitation (characteristic of such highly eccentric orbits) unless the planet was or is highly inflated and possibly had partially thermally self-disrupted from mode-based energy release. We also demonstrate that if the planet is currently assumed to reside on a near-circular orbit at $0.07 \mathrm{au}$, then non-chaotic equilibrium tides impose unrealistic values for the planet's tidal quality factor. We conclude that WD J0914+1914 b either (i) actually resides interior to $0.07 \mathrm{au}$, (ii) resembles a disrupted 'Super-Puff' whose remains reside on a circular orbit, or (iii) resembles a larger or denser ice giant on a currently eccentric orbit. Distinguishing these three possibilities strongly motivates follow-up observations.
\end{abstract}

Key words: methods: numerical - celestial mechanics-planets and satellites: detectionplanets and satellites: dynamical evolution and stability-planet-star interactions-white dwarfs.

\section{INTRODUCTION}

The first exoplanetary system signatures discovered around mainsequence stars arose from major planets (Campbell, Walker \& Yang 1988; Latham et al. 1989; Hatzes \& Cochran 1993; Mayor \& Queloz 1995; Marcy \& Butler 1996). In a twist of fate, the situation for white dwarf planetary systems is just the opposite: secure evidence for a major planet (Gänsicke et al. 2019) represents one of the last significant missing components of these systems to be found.

Over the past century (van Maanen 1917, 1919), mounting discoveries of planetary remnants in white dwarf atmospheres have led to surveys which show that 25-50 per cent of all single Milky Way white dwarfs are metal polluted with planetary materials

\footnotetext{
^E-mail: d.veras@warwick.ac.uk

$\dagger$ STFC Ernest Rutherford Fellow.
}

(Zuckerman et al. 2003, 2010; Koester, Gänsicke \& Farihi 2014). This debris is predominantly chemically consistent with fragments from rocky bodies (Zuckerman et al. 2007; Gänsicke et al. 2012; Jura \& Young 2014; Harrison, Bonsor \& Madhusudhan 2018; Hollands, Gänsicke \& Koester 2018; Doyle et al. 2019; Swan et al. 2019; Xu et al. 2019; Bonsor et al. 2020). Major rocky planets, however, are simply not numerous enough to represent the progenitors of these pollutants because they would not approach white dwarfs at a sufficiently high frequency (Veras 2016a; Veras et al. 2016).

The debris needs to arise from larger reservoirs of minor planets, such as moons (Payne et al. 2016, 2017) or analogues to the asteroid belt (Debes, Walsh \& Stark 2012; Frewen \& Hansen 2014; Antoniadou \& Veras 2016, 2019; Smallwood et al. 2018, 2019) or to the Kuiper belt (Bonsor, Mustill \& Wyatt 2011; Mustill et al. 2018; Grishin \& Veras 2019; Makarov \& Veras 2019); comets are too volatile-rich and sparse to provide as good of a match with 
the debris (Alcock, Fristrom \& Siegelman 1986; Veras, Shannon \& Gänsicke 2014a; Stone, Metzger \& Loeb 2015; Caiazzo \& Heyl 2017). However, without a stellar companion nor major planets, rocky minor planets cannot self-propel themselves into the white dwarf (Veras, Eggl \& Gänsicke 2015a; Veras, Xu \& RebassaMansergas 2018a). Despite this need for major planets at distances within tens or hundreds of au, they had remained undetected. ${ }^{1}$

Concurrent with the increasing detections of white dwarf metal pollution were the first detection of a white dwarf debris disc (Zuckerman \& Becklin 1987) and the first detection of an orbiting minor planet (Vanderburg et al. 2015), but still no major planet. Now over 40 discs are known (e.g. Gänsicke et al. 2006; Farihi 2016; Dennihy et al. 2018) and a second (Manser et al. 2019) and likely third minor planet (Vanderbosch et al. 2019; Veras, McDonald \& Makarov 2020) have been detected orbiting different white dwarfs. Nevertheless, the formation of these discs (Debes et al. 2012; Veras et al. 2014b, 2015b; Malamud \& Perets 2020a, b) still requires a major planet to perturb a minor planet into the white dwarf tidal disruption radius (Veras 2016a). Also, the origin of the near-circular orbit (Gurri, Veras \& Gänsicke 2017; Veras et al. 2017; Duvvuri et al. 2020) of the first minor planet found orbiting a white dwarf (Vanderburg et al. 2015) remains one of the foremost unexplained problems in white dwarf planetary science, but envisaging an inward migration scenario without the help of a major planet is challenging.

A breakthrough arrived when Gänsicke et al. (2019) detected robust chemical signatures of accretion from evaporation of one of these major planets around the metal-polluted and disc-bearing single white dwarf WD J0914+1914. This detection was corroborated by several factors:

(i) The chemical absence of Earth-like compositions within the atmosphere of WD J0914+1914 is highly unusual for metalpolluted white dwarfs (Xu et al. 2017; Harrison et al. 2018; Hollands et al. 2018; Doyle et al. 2019; Swan et al. 2019; Xu et al. 2019; Bonsor et al. 2020) and indicates a lack of rocky body accretion.

(ii) A pristine giant planet might be expected to contain methane in the outer layers of its atmosphere. The claim that these outer layers have been evaporated is supported by the non-detection of carbon in WD J0914+1914. Further, the upper limit implies a subsolar carbon abundance.

(iii) Orbiting WD J0914+1914 is a gas-only disc; the first to be discovered around a metal-polluted white dwarf.

(iv) The WD J0914+1914 planetary system represents the first where independent measurements of absorption lines from the atmospheric metal pollution and emission lines from the metals in the gaseous disc were both obtained and match.

As a result, we know the composition of the planet's atmosphere better than its orbit; for main-sequence planets, the opposite is nearly always true. Gänsicke et al. (2019) estimated that the current location of the planet, although still very uncertain, is at a distance of about $15 \mathrm{R}_{\odot} \approx 0.070 \mathrm{au}$. They further argued that the planet should be further away than the outer extent of the

\footnotetext{
${ }^{1}$ A near-exception includes an object residing at a distance of about 2500 au that was found orbiting WD 0806-661b and was classified as a possible brown dwarf by the authors (Luhman, Burgasser \& Bochanski 2011), despite harbouring a mass of approximately 10 Jupiter masses. Also, a different, circumbinary object of lower mass, PSR B1620-26b, was discovered orbiting both a pulsar and a white dwarf (Sigurdsson et al. 2003).
}

disc, which is constrained through emission lines to be located at about $10 \mathrm{R}_{\odot} \approx 0.046$ au. However, in principle, a sufficiently low-mass planet may be embedded within the disc and not have opened up a gap. Throughout this paper, we will keep in mind these uncertainties, but adopt 0.070 au as the current planet distance for our computations.

Although the host star has a mass of $0.56 \pm 0.03 \mathrm{M}_{\odot}$ (a common value; Tremblay et al. 2016), its cooling age of $13.3 \pm 0.5 \mathrm{Myr}$ is very young compared to most known metal-polluted white dwarfs. The term 'cooling age' simply refers to the age of the star after it became a white dwarf. For WD J0914+1914, its cooling age represents the crucial dynamical constraint. A cooling age of 13.3 Myr is considered so young because white dwarf planetary remnants have been observed in the atmospheres of white dwarfs with cooling ages of $8 \mathrm{Gyr}$ (Hollands et al. 2018). Veras \& Fuller (2019) highlighted how such young cooling ages can place constraints on the orbital history and tidal dissipation of gaseous planets.

Here, we apply their results to the WD J0914+1914 system, and henceforth use the designation WD J0914+1914 b for the planet. We address the orbital evolution of WD J0914+1914 b in Section 2, briefly discuss our results in Section 3, and conclude in Section 4 .

\section{ORBITAL EVOLUTION}

\subsection{The planet's initial position}

WD J0914+1914 b needed to survive the main sequence and giant branch stages of the star's evolution before being relocated to a distance of about $0.07 \mathrm{au}$. The planet's prospects for survival during these phases relies on a combination of its initial position (where it formed) and details of its host star evolution.

Gänsicke et al. (2019) claimed that based on the current white dwarf mass of $0.56 \pm 0.03 \mathrm{M}_{\odot}$, the star's progenitor main-sequence mass was $1.0-1.6 \mathrm{M}_{\odot}$. By considering the extremes of this range, we note that the evolution of 1.0 and $1.6 \mathrm{M}_{\odot}$ stars are qualitatively and quantitatively different. A $1.0 \mathrm{M}_{\odot}$ star features comparably significant and potentially destructive (to planets) red giant branch and asymptotic giant branch phases. Recent studies of the Solar system's post-main-sequence evolution (Schröder \& Smith 2008; Veras 2016b) revealed that the Sun's envelope will extend out to about $1 \mathrm{au}$ at the tip of both phases. In contrast, for $1.6 \mathrm{M}_{\odot}$ stars, the red giant envelope extends out to about 0.8 au (Villaver et al. 2014) whereas the asymptotic giant envelope extends out to about $1.5 \mathrm{au}$ (fig. 3 of Veras 2016a).

Numerous studies have now shown that the tidal interaction between giant branch stars and giant planets require the latter to reside at distances of at least 1 au beyond the extent of the stellar envelope in order to survive (Villaver \& Livio 2009; Kunitomo et al. 2011; Mustill \& Villaver 2012; Adams \& Bloch 2013; Villaver et al. 2014; Madappatt, De Marco \& Villaver 2016; Staff et al. 2016; Gallet et al. 2017; Rao et al. 2018; Sun et al. 2018). Therefore, regardless of the progenitor mass or the stellar model used, a reasonable assumption is that WD J0914+1914 b began the white dwarf phase at a distance of at least 2-3 au. Scenarios where the planet would instead acquire an orbital distance of just 0.07 au during a common envelope phase when the star ascends the asymptotic giant branch phase would require exceptionally finetuned migration within the stellar envelope (see section 5.3 of Campante et al. 2019), because the in-spiral time is at most $10^{4}$ orbits (MacLeod, Cantiello \& Soares-Furtado 2018). 


\subsection{Scattering the planet}

Assuming that the planet initially resides at a distance of at least 2-3 au, then it will need to be perturbed by another planet of approximately equal or greater mass to eventually reach a distance within 0.07 au. Many investigations have now shown that such inward scattering within evolved single-star multiplanet systems is feasible (Debes \& Sigurdsson 2002; Veras et al. 2013; Voyatzis et al. 2013; Mustill, Veras \& Villaver 2014; Veras \& Gänsicke 2015; Veras et al. 2016; Mustill et al. 2018; Veras et al. 2018b).

A different potential scatterer could be a stellar companion, which has been shown to easily perturb major planets in evolved planetary systems close to one of the stars (Hamers \& Portegies Zwart 2016; Stephan, Naoz \& Zuckerman 2017; Veras et al. 2017b; Stephan, Naoz \& Gaudi 2018) during the post-main-sequence phases. However, WD J0914+1914 does not have a known stellar binary companion. Further Gänsicke et al. (2019) ruled out both short-period companions and companions brighter than L5 dwarfs, the latter due to the absence of an infrared excess.

Therefore, in the absence of a detected stellar companion we conclude that WD J0914+1914 contains or recently contained (within the last $13 \mathrm{Myr}$ ) at least one other major planet. The location of these other planets depend on the details of the scattering and the dynamical instability. As the above-cited studies demonstrated, the scattering represents a delayed effect triggered by dynamical instability which is itself instigated from stellar mass loss during the giant branch phases. For WD J0914+1914, the delay could not have lasted a long time: the respective durations of the red giant phase and asymptotic giant phases of this star were, respectively, hundreds of Myr and several Myr (whereas the cooling age of WD J0914+1914 is in-between).

The scattering event needed to perturb WD J0914+1914 b into an orbit with a pericentre which is at most 0.07 au (and hence with an eccentricity of at least 0.93 ). Hence, the planet may still reside on an eccentric orbit.

An orbit which perhaps better fits the observations is one of a more circular nature. Reducing the eccentricity temporarily could be achieved with a sustained scattering scenario involving multiple planets and angular momentum transfer through the angular momentum deficit (Laskar 1997, 2000; Laskar \& Petit 2017) and secular chaos (Lithwick \& Wu 2011; Wu \& Lithwick 2011; Lithwick \& Wu 2014). However, in these scenarios, usually the innermost planet's eccentricity is permanently reduced only after tidal interactions with the star.

\subsection{Chaotic tidal evolution}

Tidal interactions between stars and planets can reduce the eccentricity of an orbit. If, during this process, angular momentum was conserved, then the quantity $a\left(1-e^{2}\right)=q(1+e)$, where $q$ is the orbital pericentre, would remain constant. Hence, the periastron distance changes by a factor of $(1+e)$ as the eccentricity changes, and circularizing from $e=1$ to $e=0$ would double $q$. This scenario illustrates that the initial orbital pericentre of WD J0914+1914 b subsequent to scattering would have been located at about $0.035 \mathrm{au}$.

What is the time-scale for this decrease in semimajor axis and increase in pericentre? Constraints on the answer may be provided by dedicated tidal studies between white dwarfs and major planets, investigations which are only starting to emerge (Veras et al. 2019; Veras \& Fuller 2019; Veras \& Wolszczan 2019). Nevertheless, with the discovery of WD J0914+1914 b, and potential for more discoveries of the same type (Schreiber et al. 2019), additional tidal studies may be warranted.

For now, we utilize the results of Veras \& Fuller (2019), which considered two types of tidal interaction between white dwarfs and, specifically, gaseous planets. The first ('chaotic tides') is when a gaseous planet experiences pericentre encounters with the white dwarf when on a very highly eccentric $(1-e \ll 0.1)$ orbit. The second ('non-chaotic tides') occurs for lower eccentricities. Like for WD J0914+1914 b, major planets orbiting white dwarfs at small distances will initially have eccentricities much higher than 0.9. Therefore, understanding and exploring whether chaotic tides 'activates' is the first task.

'Chaotic tides' is shorthand for the stochastic orbital evolution which occurs due to excitation of $\mathrm{f}$ modes within the planet and the resulting exchange of energy between those modes and the angular orbital momentum (Mardling 1995a, b; Ivanov \& Papaloizou 2004, 2007; Vick \& Lai 2018; Wu 2018; Teyssandier, Lai \& Vick 2019; Vick, Lai \& Anderson 2019). The effects of chaotic tides is drastic, sometimes allowing the semimajor axis to drop by over 90 per cent while changing the eccentricity by only a few hundredths in under 1 Myr (see e.g. fig. 1 of Veras \& Fuller 2019). Crucially, chaotic tides also produce a negligible change in the orbital pericentre.

This type of evolution would facilitate explanation of the dynamical origin of WD J0914+1914 b. However, we now argue that if the pericentre of the planet was $0.035 \mathrm{au}$, then that value renders chaotic evolution impossible unless the planet was a 'Super-Puff' (highly inflated; nomenclature from Lee \& Chiang 2016).

A criterion for initiating chaotic evolution was given in equation (28) of Vick et al. (2019), and was re-expressed in equation (13) of Veras \& Fuller (2019). We take the latter expression and simplify it $^{2}$ further to

$$
116.7 \frac{\sigma^{2} K^{2}}{\epsilon}>\left(\frac{M_{\mathrm{p}}}{M_{\star}}\right) \frac{a^{7 / 2}(1-e)^{6} \sqrt{G\left(M_{\star}+M_{\mathrm{p}}\right)}}{R_{\mathrm{p}}^{5}},
$$

where the variables on the right-hand side of the equation are standard ( $a$ for the semimajor axis, $e$ for the eccentricity, $M_{\star}$ and $M_{\mathrm{p}}$ for the mass of the star and planet, and $R_{\mathrm{p}}$ for the radius of the planet). The variables on the left-hand side are the f-mode frequency

$\epsilon=1.22 \sqrt{\frac{G M_{\mathrm{p}}}{R_{\mathrm{p}}^{3}}}$,

the spin frequency of the planet, which is assumed to rotate pseudosynchronously according to

$\Omega=\frac{1+\frac{15}{2} e^{2}+\frac{45}{8} e^{4}+\frac{5}{16} e^{6}}{\left(1-e^{2}\right)^{3 / 2}\left(1+3 e^{2}+\frac{3}{8} e^{4}\right)} \sqrt{\frac{G\left(M_{\star}+M_{\mathrm{p}}\right)}{a^{3}},}$

and

$\sigma=\epsilon+\Omega$

${ }^{2}$ For all of the detailed assumptions which enter into this criterion, see Vick et al. (2019). One of these assumptions is a value for the overlap integral, which depends on the structure of the planet. In particular, the value may be smaller for Super-Puffs - which probably have low-mass envelopes - than gas giants. We adopt the same value for the integral (0.56) as in Vick et al. (2019) and Veras \& Fuller (2019). 


\section{Boundary to initiate chaotic evolution around WD J0914+1914}

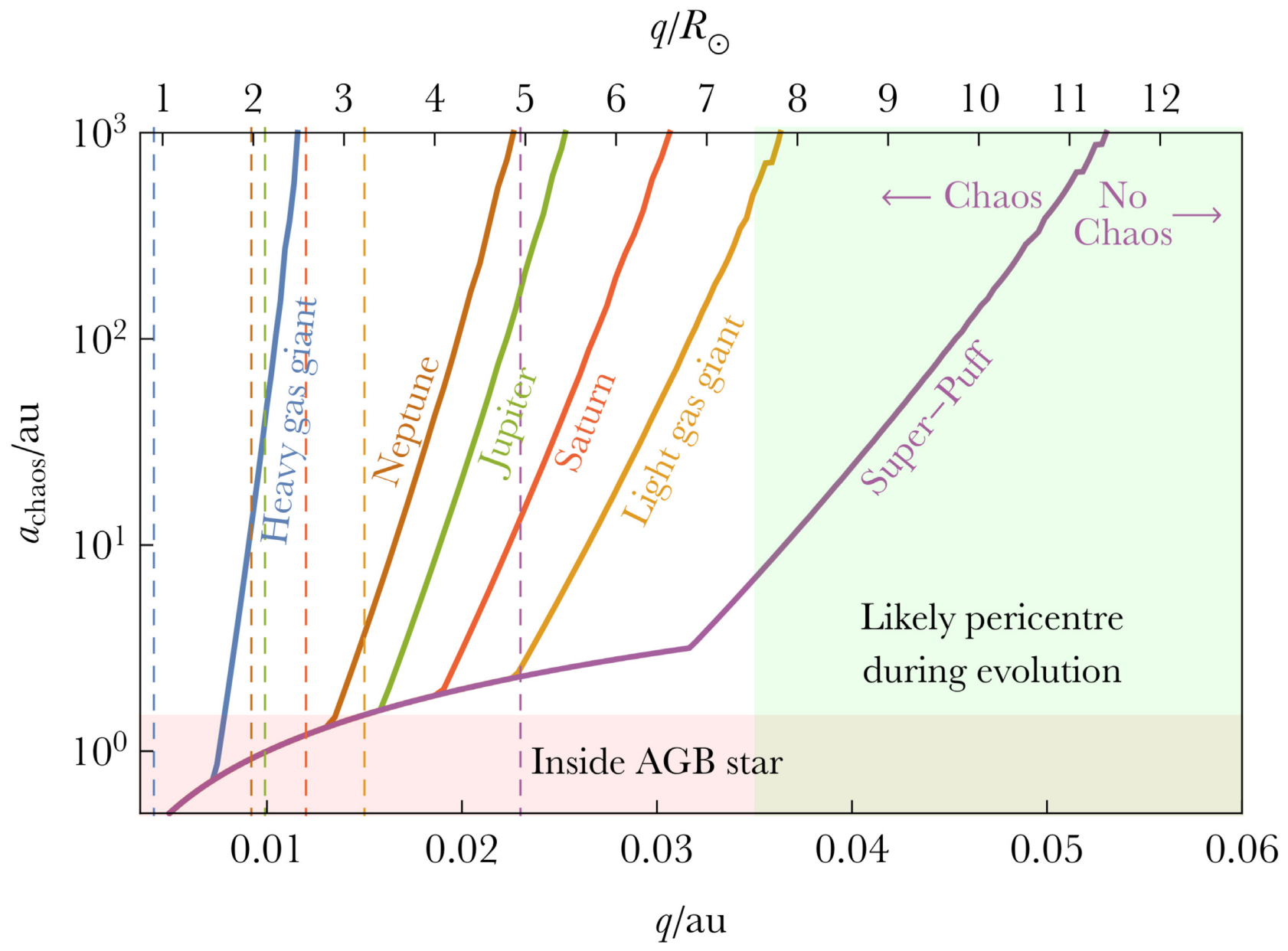

Figure 1. Demonstration that WD J0914+1914 b was very unlikely to have experienced chaotic tidal evolution unless the planet is or was a highly inflated Super-Puff. The solid curves represent the minimum initial semimajor axes ( $y$-axis) for which different types of planets would have experienced chaotic tidal evolution around the white dwarf for given orbital pericentres ( $x$-axes). The vertical dashed lines are representative white dwarf tidal disruption radii for each type of planet, ordered from left to right in the same way as the solid curves. Because Super-Puffs are particularly vunerable to self-disruption through chaotic tides, current observations may be of a partially or fully disrupted ice giant.

where

$$
\begin{aligned}
K= & 0.52 z^{3 / 2}\left[\exp \left(-\frac{2}{3} z\right)\right]\left(1-\frac{0.44}{\sqrt{z}}\right) \\
& \times\left[\frac{a(1-e)}{R_{\mathrm{p}}}\right]^{3 / 2} \sqrt{\frac{M_{\mathrm{p}}}{M_{\star}}}
\end{aligned}
$$

with

$z=\sqrt{2} \frac{\sigma}{\Omega}$.

Veras \& Fuller (2019) found that whether or not chaotic evolution is activated depends strongly on the orbital pericentre $q$, and can occur only when $u \equiv q / r_{\text {Roche }} \lesssim 2.0$, where $r_{\text {Roche }}$ is the tidal disruption radius of the white dwarf. Here, we numerically solved the set of equations (1)-(6) by computing the critical values of $a$, denoted by $a_{\text {chaos }}$, which lead to an equality in equation (1) for given values of $q$. The result is illustrated in Fig. 1.

On the figure, we computed critical curves for six different types of planets, helping to bound the entire plausible range for the initiation of chaotic tides. We assumed that the white dwarf tidal disruption radius took on the same form as in Veras \& Fuller (2019):

$r_{\text {Roche }}=1.619 R_{\odot}\left(\frac{\rho_{\mathrm{p}}}{3 \mathrm{~g} \mathrm{~cm}^{-3}}\right)^{-1 / 3}$

where $\rho_{\mathrm{p}}$ is the density of the planet. A more accurate expression for the tidal disruption radius would depend on the planet's physical characteristics, including its spin, and hence would change along with the spin after each pericentre passage. The six types of planets, presented along with their $u$ and $r_{\text {Roche values (assuming } q=0.035}$ $\mathrm{au}$ ), are

(i) An exo-Jupiter $\left(u=3.54, r_{\text {Roche }}=0.0099 \mathrm{au}\right)$.

(ii) An exo-Saturn $\left(u=2.84, r_{\text {Roche }}=0.012 \mathrm{au}\right)$.

(iii) An exo-Neptune ( $\left.u=3.80, r_{\text {Roche }}=0.0092 \mathrm{au}\right)$.

(iv) A 'heavy gas giant' $\left(M_{\mathrm{p}}=13 M_{\text {Jupiter }}\right.$ and $R_{\mathrm{p}}=R_{\text {Jupiter }}$, giving $\left.u=8.33, r_{\text {Roche }}=0.0042 \mathrm{au}\right)$.

(v) A 'light gas giant' $\left(M_{\mathrm{p}}=0.3 M_{\text {Jupiter }}\right.$ and $R_{\mathrm{p}}=R_{\text {Jupiter }}$, giving $\left.u=2.37, r_{\text {Roche }}=0.015 \mathrm{au}\right)$.

(vi) A 'Super-Puff' $\left(M_{\mathrm{p}}=4 \mathrm{M}_{\oplus}\right.$ and $R_{\mathrm{p}}=6 \mathrm{R}_{\oplus}$, giving $u=1.51$, $\left.r_{\text {Roche }}=0.023 \mathrm{au}\right)$. 
Chaotic evolution is activated only for semimajor axes above the curves. Also plotted are representative white dwarf tidal disruption radii for the different types of planets as dashed vertical lines, as well as an approximate value for the asymptotic giant branch radius for WD J0914+1914 (recall that tidal engulfment would occur at different values, again depending on the planet characteristics).

Fig. 1 clearly demonstrates that a pericentre of $0.035 \mathrm{au}$ is too high to have initiated chaotic evolution in the past unless the planet was a highly inflated 'Super-Puff'. This result also conforms with the findings from Veras \& Fuller (2019) that chaotic tides are not activated when $u \gtrsim 2.0$. For the Super-Puff case, the semimajor axis of the planet needed to be at least about 10 au to initiate chaotic tides.

Such an inflated planet would have likely experienced multiple thermalization events during the chaotic evolution. As shown in Veras \& Fuller (2019), exo-Neptune analouges would be already susceptible to self-disruption through these thermalization events. For a Super-Puff, the self-disruption would occur sooner. However, the disruption process has not yet been analysed in detail, and current observations might plausibly reveal an icy or rocky core of a disrupted planet.

\subsection{Non-chaotic tidal evolution}

If chaotic tidal evolution did not occur, then the planet's orbital and physical evolution was qualitatively different. In order to explore non-chaotic tidal evolution, we adopt the simple and standard equilibrium weak friction tidal approximation from Hut (1981).

In this approximation, the planet's semimajor axis and eccentricity evolve according to a set of differential equations which are a function of the modified quality factors of the planet and star. Veras \& Fuller (2019) found that to a good approximation for white dwarf planetary systems, dissipation in the white dwarf can be neglected and the time-scale to circularize the orbit, $\tau_{\text {circ }}$, can be empirically estimated through ${ }^{3}$

$$
\begin{aligned}
\tau_{\text {circ }} \approx & 37.4 \operatorname{Myr}\left(\frac{q}{r_{\text {Roche }}}\right)^{13 / 2}\left(\frac{Q_{\mathrm{p}}^{\prime}}{10^{6}}\right) \\
& \times\left(\frac{M_{\mathrm{p}}}{M_{\text {Jupiter }}}\right)^{-2 / 3}\left(\frac{\rho_{\mathrm{p}}}{1 \mathrm{~g} / \mathrm{cm}^{3}}\right)^{-1 / 2},
\end{aligned}
$$

where $Q_{\mathrm{p}}^{\prime}$ is the modified planetary quality factor and $\rho_{\mathrm{p}}$ is the planet's density.

Because equation (8) showcases a particularly strong dependence on $q$, we plot values of $Q_{\mathrm{p}}^{\prime}$ which yield $\tau_{\text {circ }}=13.3 \mathrm{Myr}$ (the cooling age of WD J0914+1914) as a function of $q$ in Fig. 2. The region with the green shaded background represents the likely range of $q$ values that the orbit of WD J0914+1914 b has acquired throughout white dwarf cooling. Note that regardless of the planet's $q$ value in this region, the required values of $Q_{\mathrm{p}}^{\prime}$ are typically orders of magnitude lower than the typically considered range of $10^{3}-10^{7}$ (Wu 2005; Matsumura, Peale \& Rasio 2010; Ogilvie 2014). The planet's current $q$ would have to be less than half of the estimated value of $0.07 \mathrm{au}$ in order for weakly dissipative tides to represent a more plausible circularization mechanism.

\footnotetext{
${ }^{3}$ In order to obtain the formula, they assumed a white dwarf mass of $0.60 \mathrm{M}_{\odot}$, which is sufficiently similar to the mass of WD J0914+1914 $\left(0.56 \mathrm{M}_{\odot}\right)$ for us to use here.
}

\section{DISCUSSION}

Our results suggest that a partially or fully intact WD J0914+1914 b cannot reside on a near-circular orbit of about 0.07 au unless the planet is (or was) a Super-Puff (highly inflated) which has undergone chaotic tidal evolution. Nevertheless, our understanding of tidal interactions still leaves much room for improvement. Efroimsky \& Makarov (2013) illustrated that popular tidal models are unphysical in many situations, and definitive observational confirmation of tidally induced orbital decay in extrasolar systems is only now becoming a reality (Maciejewski et al. 2013; Hoyer et al. 2016; Patra et al. 2017; Wilkins et al. 2017; Bailey \& Goodman 2019; Yee et al. 2020).

If the planet is not a Super-Puff and does have a pericentre distance of $0.07 \mathrm{au}$, then it would need to reside on an eccentric orbit; observations cannot yet support or refute this possibility. If this orbital eccentricity is sufficiently high, then it would interact repeatedly with the gaseous disc which is thought to extend out to 0.04-0.05 au. The consequences of this interaction depend on the geometric details of the disc and the relative inclination of the planet's orbit to the disc plane. The picture is complicated further by the evaporation of the planet's atmosphere, which would be a strong function of distance and be most significant close to pericentre (Schreiber et al. 2019). The potential interaction between the planet and gas disc strongly motivates follow-up observations, particularly because, as estimated earlier, the duration of pericentre passages is of the order of just days.

Instead, a partially or fully disrupted planet motivates further modelling of the energy redistribution and loss within the f modes of a planet. The destination of the energy after each thermalization event remains unknown and is dictated by the subtleties of the non-linear breaking process, which would again require detailed modelling. If the energy is assumed to be retained within the planet, then eventually the planet could self-disrupt. Even before this time, a fragile atmosphere might leak out due to only a few thermalization events.

Veras \& Fuller (2019) illustrated that the time-scale for potential self-disruption for ice giants with the same mass and radius as Neptune is less than about $10 \mathrm{Myr}$ as long as the orbital pericentre is within about $1.5 r_{\text {Roche }} \approx 0.014$ au $\approx 2 \mathrm{R}_{\odot}$. As shown above, it is theoretically difficult to circularize a planet to a semimajor axis as large as $\approx 0.07$ au (as inferred by Gänsicke et al. 2019) within the cooling age (13 Myr) of this host star. One possibility is that chaotic tidal migration of a Super-Puff planet began at smaller periastron distance, but the subsequent heating disrupted the planet (or stripped its atmosphere) to produce the debris disc around WD J0914+1914. Another possibility is that the core of such a partially disrupted planet could remain intact and circularize to a small semimajor axis upon interaction with the disruption debris.

A third possibility is that the current orbit and physical state of WD J0914+1914 b is the result of a giant impact between two major planets; the white dwarf, being so young, could have easily harboured a violent dynamical environment. This impact which may have occurred around $0.07 \mathrm{au}$ - could have reduced the eccentricity of the planet's orbit, as well as produced a stream of debris. The high luminosity of this newly formed white dwarf could have then generated an inward drag of the resulting debris (Stone et al. 2015; Veras et al. 2015b). Over time, in combination with evaporation from the planet's atmosphere, the interaction between the planet and the debris may have cleared out the region in-between the disc and planet (between about 0.045 and $0.070 \mathrm{au})$. 


\section{Modified tidal quality function required to circularize}

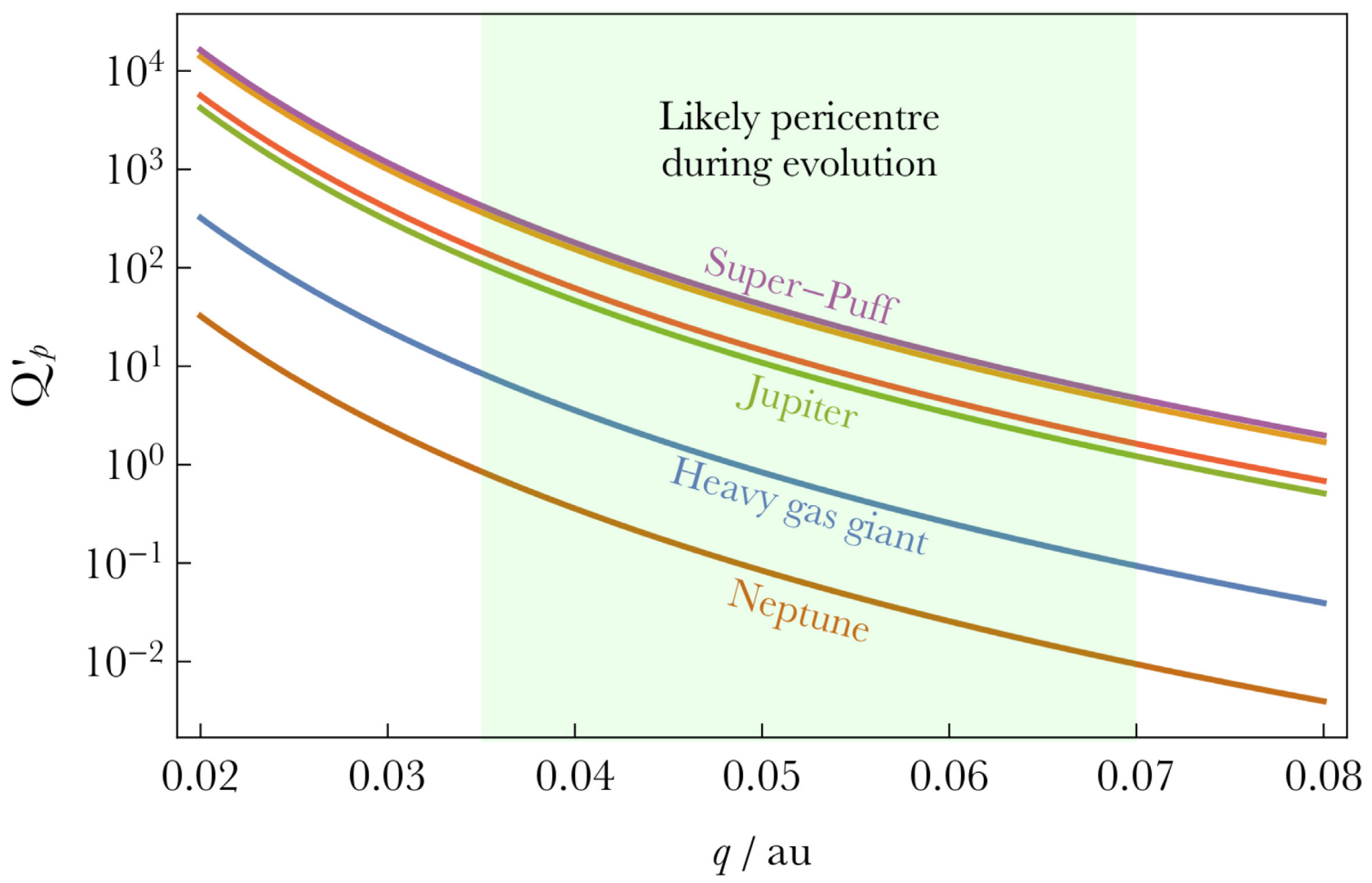

Figure 2. Modified tidal quality factors ( $y$-axis) required to circularize the orbit of WD J0914+1914 b depending on its physical parameters (various curves) as a function of the orbital pericentre ( $x$-axis). Potential values of the tidal quality factors are unrealistically small within the green shaded region, which represents the likely range of $q$ values that the planet has acquired during white dwarf cooling if the planet's current pericentre resides at 0.07 au (as assumed in Gänsicke et al. 2019).

A final possibility is that the semimajor axis of WD J0914+1914 b is smaller than the 0.07 au estimated by Gänsicke et al. (2019). If the planet's mass is less than about $20 \mathrm{M}_{\oplus}$, then it may not be massive enough to open a gap or truncate the accretion disc. Hence, the planet could orbit within the debris disc or at its outer edge with a semimajor axis of $\approx 0.04$ au. In this scenario, the initial periastron distance of the planet may have been closer to 0.02 $\mathrm{au}$, increasing the plausibility of both the chaotic and equilibrium tidal circularization phases. A prediction of this model could be tested through periodic distortions in the shape of the emission lines of the disc, similar to those observed in the debris disc around SDSS J1228+1040 (Manser et al. 2019).

Despite the fine details of the planet's current orbit, the planet's classification as a Super-Puff would be consistent with the gravitational scattering hypothesis. The planet could have been born at a sufficiently large semimajor axis under a wide range of nebular conditions (Lee, Chiang \& Ferguson 2018) to retain its puffy atmosphere throughout stellar evolution (Wang \& Dai 2019). Subsequently, the planet may have migrated inward (Lee \& Chiang 2016) under the high-eccentricity regime to arrive at its current location.

\section{SUMMARY}

The robust signatures of the first major planet found orbiting a single white dwarf (Gänsicke et al. 2019) reveal a planet which is better constrained chemically than dynamically. Observations strongly suggest that the planet is an ice giant (or the remnants of one), but its mass, radius, and orbit are unknown, except for an inferred but still uncertain current distance of about $0.07 \mathrm{au}$. Contrastingly, the cooling age of the planet's host star is wellconstrained (13.3 $\pm 0.5 \mathrm{Myr})$ and in fact is much better constrained than the age of almost any other known major planet host star.

The juxtaposition of such robust and poor constraints requires an unorthodox analysis to identify the dynamical history of WD J0914+1914 b. Here, we considered scenarios which can explain the observations by assuming the inferred current distance of $0.07 \mathrm{au}$. We claim that the planet must have resided at a distance of at least a few au at the onset of the white dwarf phase, and subsequently been scattered towards the white dwarf with a pericentre which is about $0.035 \mathrm{au}$. This scattering event requires the current or former presence of at least one other major planet in the system of comparable or greater mass.

We also suggest that tidal circularization at 0.07 au could not have occurred unless the planet is a highly inflated 'Super-Puff'. In the distance range $0.035-0.070 \mathrm{au}$, weak equilibrium tides require unrealistically low values for the planetary quality factor to circularize an orbit within the white dwarf's cooling age of $13 \mathrm{Myr}$. Instead, chaotic f-mode tidal evolution would be required, which could shrink the semimajor axis orders of magnitude more quickly.

Further, the inflated nature of this planet implies, from Veras \& Fuller (2019), that the planet would have experienced thermalization events during this chaotic tidal evolution. These events could have 
partially or fully disrupted the planet. Alternatively, a denser or larger ice giant may be intact and passing through 0.07 au on its way to a much lower pericentre. Finally, a remaining possibility is that an intact planet orbits within the debris disc at $\approx 0.04$ au, allowing for tidal circularization to deliver the planet to its current location within the short cooling age of the white dwarf.

These varied and violent possibilities provide strong motivation for the acquisition of future observational data of WD J0914+1914 b.

\section{ACKNOWLEDGEMENTS}

We thank the referee for helpful comments which have improved the manuscript, and also thank Uri Malamud and Alexander Stephan for useful discussions. DV gratefully acknowledges the support of the STFC via an Ernest Rutherford Fellowship (grant ST/P003850/1). JF acknowledges support from an Innovator Grant from The Rose Hills Foundation and the Sloan Foundation through grant FG-201810515.

\section{REFERENCES}

Adams F. C., Bloch A. M., 2013, ApJL, 777, L30

Alcock C., Fristrom C. C., Siegelman R., 1986, ApJ, 302, 462

Antoniadou K. I., Veras D., 2016, MNRAS, 463, 4108

Antoniadou K. I., Veras D., 2019, A\&A, 629, A126

Bailey A., Goodman J., 2019, MNRAS, 482, 1872

Bonsor A., Mustill A. J., Wyatt M. C., 2011, MNRAS, 414, 930

Bonsor A., Carter P. J., Hollands M., Gänsicke B. T., Leinhardt Z., Harrison J. H. D. 2020, MNRAS, 492, 2683

Caiazzo I., Heyl J. S., 2017, MNRAS, 469, 2750

Campante T. L. et al., 2019, ApJ, 885, 31

Campbell B., Walker G. A. H., Yang S., 1988, ApJ, 331, 902

Debes J. H., Sigurdsson S., 2002, ApJ, 572, 556

Debes J. H., Walsh K. J., Stark C., 2012, ApJ, 747, 148

Dennihy E., Clemens J. C., Dunlap B. H., Fanale S. M., Fuchs J. T., Hermes J. J., 2018, ApJ, 854, 40

Doyle A. E., Young E. D., Klein B., Zuckerman B., Schlichting H. E., 2019, Science, 366, 356

Duvvuri G., Redfield S., Veras D., 2020, AAS Journals, submitted

Efroimsky M., Makarov V. V., 2013, ApJ, 764, 26

Farihi J., 2016, New Astron. Rev., 71, 9

Frewen S. F. N., Hansen B. M. S., 2014, MNRAS, 439, 2442

Gallet F., Bolmont E., Mathis S., Charbonnel C., Amard L., 2017, A\&A, 604, A112

Gänsicke B. T., Marsh T. R., Southworth J., Rebassa-Mansergas A., 2006, Science, 314, 1908

Gänsicke B. T., Koester D., Farihi J., Girven J., Parsons S. G., Breedt E., 2012, MNRAS, 424, 333

Gänsicke B. T., Schreiber M. R., Toloza O., Gentile Fusilo N. P., Koester D., Manser C. J., 2019, Nature, 576, 61-64

Grishin E., Veras D., 2019, MNRAS, 489, 168

Gurri P., Veras D., Gänsicke B. T., 2017, MNRAS, 464, 321

Hamers A. S., Portegies Zwart S. F., 2016, MNRAS, 462, L84

Harrison J. H. D., Bonsor A., Madhusudhan N., 2018, MNRAS, 479, 3814.

Hatzes A. P., Cochran W. D., 1993, ApJ, 413, 339

Hollands M. A., Gänsicke B. T., Koester D., 2018, MNRAS, 477, 93.

Hoyer S., Pallé E., Dragomir D., Murgas F., 2016, AJ, 151, 137

Hut P., 1981, A\&A, 99, 126

Ivanov P. B., Papaloizou J. C. B., 2004, MNRAS, 347, 437

Ivanov P. B., Papaloizou J. C. B., 2007, A\&A, 476, 121.

Jura M., Young E. D., 2014, Annu. Rev. Earth. Planet. Sci., 42, 45

Koester D., Gänsicke B. T., Farihi J., 2014, A\&A, 566, A34

Kunitomo M., Ikoma M., Sato B., Katsuta Y., Ida S., 2011, ApJ, 737, 66

Laskar J., 1997, A\&A, 317, L75

Laskar J., 2000, Phys. Rev. Lett., 84, 3240
Laskar J., Petit A. C., 2017, A\&A, 605, A72

Latham D. W., Mazeh T., Stefanik R. P., Mayor M., Burki G., 1989, Nature, 339,38

Lee E. J., Chiang E., 2016, ApJ, 817, 90

Lee E. J., Chiang E., Ferguson J. W., 2018, MNRAS, 476, 2199

Lithwick Y., Wu Y., 2011, ApJ, 739, 31

Lithwick Y., Wu Y., 2014, PNAS, 111, 12610

Luhman K. L., Burgasser A. J., Bochanski J. J., 2011, ApJL, 730, L9

Maciejewski G. et al., 2013, A\&A, 551, A108

MacLeod M., Cantiello M., Soares-Furtado M., 2018, ApJL, 853, L1

Madappatt N., De Marco O., Villaver E., 2016, MNRAS, 463, 1040

Makarov V. V., Veras D., 2019, ApJ, 886, 127

Malamud U., Perets H., 2020a, MNRAS, submitted

Malamud U., Perets H., 2020b, MNRAS, submitted

Manser C. J. et al., 2019, Science, 364, 66

Marcy G. W., Butler R. P., 1996, ApJL, 464, L147

Mardling R. A., 1995a, ApJ, 450, 722

Mardling R. A., 1995b, ApJ, 450, 732

Matsumura S., Peale S. J., Rasio F. A., 2010, ApJ, 725, 1995

Mayor M., Queloz D., 1995, Nature, 378, 355

Mustill A. J., Villaver E., 2012, ApJ, 761, 121

Mustill A. J., Veras D., Villaver E., 2014, MNRAS, 437, 1404

Mustill A. J., Villaver E., Veras D., Gänsicke B. T., Bonsor A., 2018, MNRAS, 476, 3939

Ogilvie G. I., 2014, ARA\&A, 52, 171

Patra K. C., Winn J. N., Holman M. J., Yu L., Deming D., Dai F., 2017, AJ, 154,4

Payne M. J., Veras D., Holman M. J., Gänsicke B. T., 2016, MNRAS, 457, 217

Payne M. J., Veras D., Gänsicke B. T., Holman M. J., 2017, MNRAS, 464, 2557

Rao S., Meynet G., Eggenberger P., Haemmerlé L., Privitera G., Georgy C., Ekström S., Mordasini C., 2018, A\&A, 618, A18

Schreiber M. R., Gänsicke B. T., Toloza O., Hernandez M.-S., Lagos F., 2019, ApJL, 887, L4

Schröder K.-P., Smith R., 2008, MNRAS, 386, 155

Sigurdsson S., Richer H. B., Hansen B. M., Stairs I. H., Thorsett S. E., 2003, Science, 301, 193

Smallwood J. L., Martin R. G., Livio M., Lubow S. H., 2018, MNRAS, 480, 57

Smallwood J. L. et al., 2019, MNRAS, submitted

Staff J. E., De Marco O., Wood P., Galaviz P., Passy J.-C., 2016, MNRAS, 458,832

Stephan A. P., Naoz S., Zuckerman B., 2017, ApJL, 844, L16

Stephan A. P., Naoz S., Gaudi B. S., 2018, AJ, 156, 128

Stone N., Metzger B. D., Loeb A., 2015, MNRAS, 448, 188

Sun M., Arras P., Weinberg N. N., Troup N. W., Majewski S. R., 2018, MNRAS, 481, 4077

Swan A., Farihi J., Koester D., Hollands M., Parsons S., Cauley P. W., Redfield S., Gänsicke B. T., 2019, MNRAS, 490, 202

Teyssandier J., Lai D., Vick M., 2019, MNRAS, 486, 2265

Tremblay P.-E., Cummings J., Kalirai J. S., Gänsicke B. T., Gentile-Fusillo N., Raddi R., 2016, MNRAS, 461, 2100

van Maanen A., 1917, PASP, 29, 258

van Maanen A., 1919, AJ, 32, 86

Vanderbosch Z. et al., 2019, preprint (arXiv:1908.09839)

Vanderburg A. et al., 2015, Nature, 526, 546

Veras D., 2016a, R. Soc. Open Sci., 3, 150571

Veras D., 2016b, MNRAS, 463, 2958

Veras D., Fuller J., 2019, MNRAS, 489, 2941

Veras D., Gänsicke B. T., 2015, MNRAS, 447, 1049

Veras D., Wolszczan A., 2019, MNRAS, 488, 153

Veras D., Mustill A. J., Bonsor A., Wyatt M. C., 2013, MNRAS, 431, 1686

Veras D., Shannon A., Gänsicke B. T., 2014a, MNRAS, 445, 4175

Veras D., Leinhardt Z. M., Bonsor A., Gänsicke B. T., 2014b, MNRAS, 445, 2244

Veras D., Eggl S., Gänsicke B. T., 2015a, MNRAS, 452, 1945 
Veras D., Leinhardt Z. M., Eggl S., Gänsicke B. T., 2015b, MNRAS, 451, 3453

Veras D., Mustill A. J., Gänsicke B. T., Redfield S., Georgakarakos N., Bowler A. B., Lloyd M. J. S., 2016, MNRAS, 458, 3942

Veras D., Carter P. J., Leinhardt Z. M., Gänsicke B. T., 2017, MNRAS, 465, 1008

Veras D., Georgakarakos N., Dobbs-Dixon I., Gänsicke B. T., 2017b, MNRAS, 465, 2053

Veras D., Xu S., Rebassa-Mansergas A., 2018a, MNRAS, 473, 2871

Veras D., Georgakarakos N., Gänsicke B. T., Dobbs-Dixon I., 2018b, MNRAS, 481, 2180

Veras D. et al., 2019, MNRAS, 486, 3831

Veras D., McDonald C. H., Makarov V. V., 2020, MNRAS, preprint (arXiv: 2001.08223)

Vick M., Lai D., 2018, MNRAS, 476, 482

Vick M., Lai D., Anderson K. R., 2019, MNRAS, 484, 5645

Villaver E., Livio M., 2009, ApJL, 705, L81

Villaver E., Livio M., Mustill A. J., Siess L., 2014, ApJ, 794, 3

Voyatzis G., Hadjidemetriou J. D., Veras D., Varvoglis H., 2013, MNRAS, 430, 3383

Wang L., Dai F., 2019, ApJL, 873, L1
Wilkins A. N., Delrez L., Barker A. J., Deming D., Hamilton D., Gillon M., Jehin E., 2017, ApJ, 836, L24

Wu Y., 2005, ApJ, 635, 688

Wu Y., 2018, AJ, 155, 118

Wu Y., Lithwick Y., 2011, ApJ, 735, 109

Xu S., Zuckerman B., Dufour P., Young E. D., Klein B., Jura M., 2017, ApJ, 836, L7

Xu S., Dufour P., Klein B., Melis C., Monson N. N., Zuckerman B., Young E. D., Jura M. A., 2019, AJ, 158, 242

Yee S. W. et al., 2020, ApJ, 888, L5

Zuckerman B., Becklin E. E., 1987, Nature, 330, 138

Zuckerman B., Koester D., Reid I. N., Hünsch M., 2003, ApJ, 596, 477

Zuckerman B., Koester D., Melis C., Hansen B. M., Jura M., 2007, ApJ, 671,872

Zuckerman B., Melis C., Klein B., Koester D., Jura M., 2010, ApJ, 722, 725

This paper has been typeset from a $\mathrm{T}_{\mathrm{E}} \mathrm{X} / \mathrm{LT}_{\mathrm{E}} \mathrm{X}$ file prepared by the author. 